\title{
A Rare Twist of the Forgotten Disease: A Case of Fusobacterium necrophorum Sepsis with Portomesenteric Thrombosis and a Review of the Literature
}

\author{
Nicholas Lazar, ${ }^{1}$ Kamil Sardarli, ${ }^{1}$ Zaid Imam $\mathbb{D}^{\mathrm{D}},{ }^{2}$ Majd Khasawneh, ${ }^{3}$ and Ismail Hader ${ }^{4}$ \\ ${ }^{1}$ Department of Internal Medicine, Beaumont Health, Royal Oak, MI, USA \\ ${ }^{2}$ Division of Gastroenterology and Hepatology, Department of Internal Medicine, Beaumont Health, Royal Oak, MI, USA \\ ${ }^{3}$ Division of Pulmonary Medicine and Critical Care, Department of Internal Medicine, University of Florida, Gainesville, FL, USA \\ ${ }^{4}$ Division of Gastroenterology and Hepatology, Sunrise Health Graduate Medical Education (GME), Las Vegas, NV, USA
}

Correspondence should be addressed to Zaid Imam; zaid.imam@beaumont.org

Received 7 December 2020; Accepted 22 May 2021; Published 28 May 2021

Academic Editor: Chia-Tung Shun

Copyright ( $\odot 2021$ Nicholas Lazar et al. This is an open access article distributed under the Creative Commons Attribution License, which permits unrestricted use, distribution, and reproduction in any medium, provided the original work is properly cited.

\begin{abstract}
Abdominal variants of Lemierre's syndrome presenting with pylephlebitis are rare, and the role of anticoagulation in treatment is controversial. We hereby report a case of pylephlebitis secondary to F. necrophorum bacteremia in a 57-year-old female originating from bacterial translocation secondary to colitis, who developed a favorable outcome with prompt treatment with antibiotics and anticoagulation. We also perform a literature review on similar cases in the literature and discuss management options of this rare but potentially fatal complication.
\end{abstract}

\section{Introduction}

Lemierre's syndrome, known as the "forgotten disease" is defined by septic thrombophlebitis of the internal jugular vein in the setting of Fusobacterium necrophorum infection. Pylephlebitis or septic thrombophlebitis of the portal venous system is a rare complication of appendicitis, cholecystitis, pancreatitis, diverticulitis, and other intra-abdominal infections [1]. It presents with fever, abdominal pain, nausea, and vomiting and carries a mortality ranging between $25-80 \%$, and early recognition is key to improved outcomes [1-4]. Abdominal variants of Lemierre's syndrome with pylephlebitis are exceedingly rare and present management controversies, particularly regarding anticoagulation $[4,5]$. Hereby, we report a case of pylephlebitis secondary to F. necrophorum bacteremia and review relevant literature on this complication.

\section{Case Report}

A 57-year-old female with prior history of nonischemic cardiomyopathy and alcohol abuse was admitted to our facility with a three-day history of myalgias and chills. Accompanied by her husband, he corroborated that she became increasingly confused over the same duration. She had no prior history of cirrhosis or chronic pancreatitis. A review of systems was otherwise negative. She had no recent dental work or procedures. On exam, she was ill-appearing with no abdominal tenderness or guarding, a normal cardiopulmonary examination, and negative meningeal signs. She was febrile to $39.1^{\circ} \mathrm{C}$ $\left(102.4^{\circ} \mathrm{F}\right)$, tachycardiac to 113 beats/minute, had a blood pressure of 113/80 $\mathrm{mmHg}$, and was saturating well on room air.

Initial labs revealed a neutrophil count of 9,100 cells $/ \mathrm{mm}$ [3], platelet count of $91,000 \mathrm{cells} / \mathrm{mm}$ [3], and lactic acid of 1.0 (normal: <2) mmol/L. Mild elevations in her aspartate aminotransferase (AST) and alanine aminotransferase to 55 (normal: 10-37) U/L and 62 (normal: 8-37) U/L, respectively, were noted with a normal alkaline phosphatase level of 58 (normal: 30-110) U/L. A chest X-ray and urinalysis were normal, and blood cultures were obtained. The patient rapidly deteriorated, became hypotensive and was resuscitated with isotonic fluid boluses, started on broad spectrum antibiotics with intravenous vancomycin and meropenem given a prior history of allergy to penicillin, and admitted to the intensivecare unit. 
On her second day of admission, her mentation improved but she developed profuse, watery diarrhea and lower abdominal pain. Clostridium difficile testing and stool cultures were obtained and were negative. A computed tomography of her abdomen and pelvis with intravenous and oral contrast showed mild sigmoid colitis but no definite evidence of diverticulitis and cholelithiasis with no pericholecystic fluid (Figure 1). On day 2 of admission, the blood cultures returned positive for a Gram-negative bacillus identified on day 4 as F. necrophorum. A progressive elevation of her liver chemistries to ALP of $415 \mathrm{U} / \mathrm{L}$, AST of $154 \mathrm{U} / \mathrm{L}$, ALT of $134 \mathrm{U} / \mathrm{L}$, and total bilirubin of $1.5 \mathrm{mg} / \mathrm{dl}$ prompted evaluation with a magnetic resonance pancreaticography (MRCP) for a biliary source of infection. Her common bile duct was normal in diameter at $7 \mathrm{~mm}$ with no filling defects, no extra- or intrahepatic biliary duct dilatation, and no pericholecystic inflammation or evidence of pancreatitis noted. However, the MRCP identified nonocclusive thrombosis of the proximal superior mesenteric vein and a few right portal vein branches with adjacent impaired hepatic perfusion. Given these findings and concern for focal hepatic tissue infarction, anticoagulation with intravenous heparin was initiated for a total of 72 hours titrated to a goal activated partial thromboplastin time (aPTT) of 60-80 seconds before transitioning to apixaban $5 \mathrm{mg}$ twice daily.

With continued supportive care and antibiotic therapy, the patient improved gradually, her symptoms resolved, and she was discharged from our facility to complete a 14-day course of ertapenem. Additionally, she was treated with apixaban $5 \mathrm{mg}$ twice daily for 3 months for her portomesenteric thrombosis. Her treatment course was uneventful, and no bleeding events occurred.

To date, the reported patient had two subsequent episodes of sigmoid diverticulitis 1 year later, the latter of which was complicated by perforation requiring a lower anterior resection, ileostomy placement, and subsequent reversal. She recovered well from her operations and has had no recurrent portal, mesenteric, or venous thromboses.

\section{Discussion}

Fusobacterium necrophorum is a commensal Gram-negative bacillus colonizing the respiratory, gastrointestinal, and female genital tract [6] and implicated as the causative agent of Lemierre's syndrome manifesting with septic internal jugular vein thrombophlebitis and often with septic pulmonary emboli $[6,7]$. Pylephlebitis, or suppurative thrombophlebitis of the portomesenteric venous system, is a rare complication of intra-abdominal infections and is hardly ever a sequela of Fusobacterium spp. septicemia [4]. We hereby report a case of septic portomesenteric thrombosis secondary to Fusobacterium necrophorum bacteremia in the setting of sigmoid colitis to add to a total of 21 cases of Fusobacterium-spp.-infection-related pylephlebitis reported in the literature [8-27].

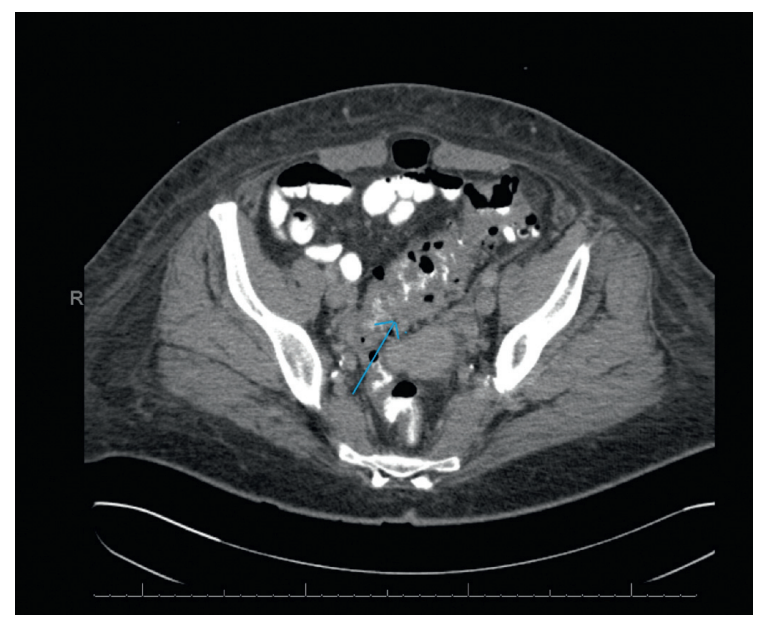

FIgURE 1: Axial computed tomography of the abdomen and pelvis demonstrating sigmoid colonic thickening and mild sigmoid colitis with no evidence of perforation on day 2 of hospitalization (blue arrow).

Clinically, pylephlebitis presents with fever, abdominal pain, hepatosplenomegaly, and, less commonly, ascites [4]. Neutrophilic leukocytosis and liver chemistry disturbances are commonly encountered in pylephlebitis from all bacterial etiologies [3, 4]. In reported cases of Fusobacterium-associated pylephlebitis (Table 1); liver enzyme abnormalities occurred in $16(72.7 \%)$ patients, including the reported case. The median patient age in Fusobacterium-spp.-associated pylephlebitis was 52 years (interquartile range (IQR): 36-63) similar to a case series of patients with pylephlebitis from other bacterial etiologies and the age of the reported patient [5].

In Fusobacterium-spp.-associated pylephlebitis, hepatic abscesses (27\%) and oropharyngeal infections (13.6\%) represented the most common identified primary infection site, and no clear primary infection site was identified in $36.4 \%$ of patients (Table 1 ). This contrasts with pylephlebitis from other bacterial etiologies whereby diverticulitis, pancreaticobiliary etiologies, and intra-abdominal abscesses represent the most common identified primary infection sites $[4,5]$. In the reported case, bacteremia from sigmoid colitis represents the likely source of infection in the absence of an alternative site and the patient's diarrhea.

Recent or remote abdominal surgery and immunosuppression have been reported as associated conditions with the development of pylephlebitis; however, pylephlebitis can occur in previously healthy individuals as well, highlighting the importance of a high index of suspicion in patients with intra-abdominal pathologies or Fusobacterium spp. bacteremia [3, 9, 12, 13, 15, 16, 22]. Diagnosis of pylephlebitis is based on confirmatory imaging findings of portal vein or mesenteric vein thrombosis in the setting of systemic infection. Intravenous contrast-enhanced computerized tomography (CT), ultrasonography, or magnetic resonance imaging (MRI) can all be used to establish the diagnosis, with the former reported as the modality of choice given its availability and high sensitivity [28]. 
TABLE 1: Demographic and clinical characteristics and diagnostic imaging modalities in patients with Fusobacterium-spp.-associated pylephlebitis.

\begin{tabular}{|c|c|c|c|c|c|c|c|c|c|}
\hline Author (year) & $\begin{array}{l}\text { Age, } \\
\text { Isex }\end{array}$ & Comorbidities & Symptoms & $\begin{array}{c}\text { Bilirubin } \\
(\mathrm{mg} / \mathrm{dl})\end{array}$ & $\begin{array}{l}\text { ALP } \\
(\mathrm{U} / \mathrm{L}) \\
\end{array}$ & $\begin{array}{l}\text { AST } \\
(\mathrm{U} / \mathrm{L}) \\
\end{array}$ & $\begin{array}{l}\text { ALT } \\
(\mathrm{U} / \mathrm{L}) \\
\end{array}$ & $\begin{array}{l}\text { Source of } \\
\text { infection }\end{array}$ & $\begin{array}{l}\text { Imaging } \\
\text { modality }\end{array}$ \\
\hline Presented case & $57, \mathrm{~F}$ & $\begin{array}{l}\text { EtOH abuse and } \\
\text { nonischemic CM }\end{array}$ & $\begin{array}{l}\text { Fever, myalgias, } \\
\text { and confusion } \\
\text { EtOH abuse }\end{array}$ & 1.4 & 348 & 124 & 104 & Colitis & MRCP \\
\hline $\begin{array}{l}\text { Abdallah et al. } \\
(2020)\end{array}$ & $37, \mathrm{M}$ & Migraines & $\begin{array}{c}\text { fever, diarrhea, } \\
\text { and abdominal } \\
\text { pain }\end{array}$ & 2.7 & 174 & 152 & 167 & Diverticulitis & CT \\
\hline $\begin{array}{l}\text { Hamera et al. } \\
(2019)\end{array}$ & $51, \mathrm{M}$ & $\begin{array}{l}\text { UC, T2DM, and } \\
\text { COPD }\end{array}$ & $\begin{array}{l}\text { Fever and } \\
\text { weakness }\end{array}$ & 1.6 & - & 45 & 112 & Cholangitis & $\begin{array}{l}\text { US and } \\
\text { CT }\end{array}$ \\
\hline $\begin{array}{l}\text { Le Roux et al. } \\
(2006)\end{array}$ & $43, \mathrm{M}$ & $\begin{array}{l}\text { EtOH abuse and } \\
\text { pancreatitis }\end{array}$ & $\begin{array}{c}\text { Fever and } \\
\text { myalgias }\end{array}$ & NL & NL & NL & NL & No source identified & $\begin{array}{l}\text { US and } \\
\text { CT }\end{array}$ \\
\hline $\begin{array}{l}\text { Mellor et al. } \\
(2017)\end{array}$ & $64, \mathrm{M}$ & None & $\begin{array}{l}\text { Fever, diarrhea, } \\
\text { and abdominal } \\
\text { pain }\end{array}$ & 3.4 & 234 & - & - & $\begin{array}{l}\text { Diverticulitis and } \\
\text { pericolonic abscess }\end{array}$ & CT \\
\hline $\begin{array}{l}\text { Moore et al. } \\
(2016)\end{array}$ & $60, M$ & Not specified & $\begin{array}{l}\text { Fever, epigastric } \\
\text { pain, and weight } \\
\text { loss }\end{array}$ & NL & NL & $\mathrm{NL}$ & NL & Unclear source & CT \\
\hline $\begin{array}{l}\text { Radovanovic } \\
\text { et al. (2019) }\end{array}$ & $69, \mathrm{M}$ & HNSCC & $\begin{array}{c}\text { Fever and } \\
\text { abdominal pain }\end{array}$ & - & 300 & - & - & Liver abscess & $\begin{array}{l}\text { CT and } \\
\text { MRI }\end{array}$ \\
\hline $\begin{array}{l}\text { Rahmati et al. } \\
\text { (2017) }\end{array}$ & $59, \mathrm{~F}$ & Multiple sclerosis & $\begin{array}{l}\text { Abdominal pain, } \\
\text { fatigue, and } \\
\text { weight loss }\end{array}$ & 1.6 & 264 & 47 & 41 & Hepatic abscess & $\begin{array}{l}\text { CT and } \\
\text { MRI }\end{array}$ \\
\hline $\begin{array}{l}\text { Tharu and et al } \\
(2020)\end{array}$ & $41, M$ & Diverticulosis & $\begin{array}{c}\text { Fever and } \\
\text { diarrhea }\end{array}$ & - & 152 & - & - & $\begin{array}{c}\text { Hepatic and brain } \\
\text { abscesses }\end{array}$ & CT \\
\hline $\begin{array}{l}\text { Akhrass et al. } \\
(2015)\end{array}$ & $32, \mathrm{M}$ & None & Epigastric pain & NL & NL & $\mathrm{NL}$ & NL & $\begin{array}{l}\text { Acute perforated } \\
\text { appendicitis }\end{array}$ & $\begin{array}{l}\text { US and } \\
\text { CT }\end{array}$ \\
\hline $\begin{array}{l}\text { Zheng et al. } \\
(2014)\end{array}$ & $73, \mathrm{M}$ & $\begin{array}{l}\text { Hypertension, T2DM, } \\
\text { and CAD }\end{array}$ & $\begin{array}{c}\text { Fever and } \\
\text { epigastric pain }\end{array}$ & NL & NL & NL & NL & No identified source & CT \\
\hline $\begin{array}{l}\text { Hamidi et al. } \\
(2008) \text { case } 1\end{array}$ & $23, \mathrm{M}$ & None & $\begin{array}{l}\text { Fever, epigastric } \\
\text { pain, diarrhea, } \\
\text { and jaundice }\end{array}$ & 9.4 & 528 & - & - & No identified source & YS \\
\hline $\begin{array}{l}\text { Hamidi et al. } \\
(2008) \text { case } 2\end{array}$ & $41, \mathrm{M}$ & EtOH abuse & $\begin{array}{l}\text { Fever, abdominal } \\
\text { pain, and } \\
\text { jaundice }\end{array}$ & 5.3 & - & 254 & 59 & No identified source & CT \\
\hline Soo et al. (1999) & $31, \mathrm{M}$ & None & $\begin{array}{l}\text { Fever, abdominal } \\
\text { pain, diarrhea, } \\
\text { and jaundice }\end{array}$ & 5.9 & 295 & 79 & 133 & No identified source & $\begin{array}{l}\text { US and } \\
\text { MRI }\end{array}$ \\
\hline $\begin{array}{l}\text { Shahani et al. } \\
\text { (2011) }\end{array}$ & $34, \mathrm{M}$ & $\begin{array}{l}\text { EtOH abuse and } \\
\text { chronic pancreatitis }\end{array}$ & Epigastric pain & $\mathrm{NL}$ & $\mathrm{NL}$ & NL & $\mathrm{NL}$ & $\begin{array}{l}\text { Hepatic, pancreatic, } \\
\text { and splenic abscesses }\end{array}$ & CT \\
\hline $\begin{array}{l}\text { Clarke et al. } \\
(2003)\end{array}$ & $19, \mathrm{~F}$ & Previously healthy & $\begin{array}{l}\text { Fever, abdominal } \\
\text { pain, and } \\
\text { jaundice }\end{array}$ & 5.7 & 331 & - & 52 & Hepatic abscesses & $\begin{array}{l}\text { US and } \\
\text { CT }\end{array}$ \\
\hline $\begin{array}{l}\text { Redford et al. } \\
\text { (2005) }\end{array}$ & $53, \mathrm{M}$ & Previously healthy & $\begin{array}{l}\text { Fever, abdominal } \\
\text { pain, and } \\
\text { vomiting }\end{array}$ & 1.4 & 194 & - & - & No identified source & CT \\
\hline $\begin{array}{l}\text { Bultink et al. } \\
\text { (1999) }\end{array}$ & $23, \mathrm{M}$ & $\begin{array}{l}\text { Previously healthy and } \\
\text { oropharyngeal } \\
\text { infection } 5 \text { weeks prior }\end{array}$ & $\begin{array}{l}\text { Fever, abdominal } \\
\text { pain, and } \\
\text { vomiting }\end{array}$ & - & 192 & - & 113 & Possible pharyngitis & $\begin{array}{l}\text { US and } \\
\text { CT }\end{array}$ \\
\hline $\begin{array}{l}\text { Verna et al. } \\
(2004)\end{array}$ & $56, \mathrm{M}$ & 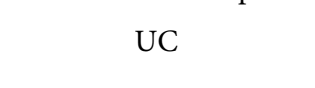 & $\begin{array}{l}\text { Fever, anorexia, } \\
\text { and jaundice }\end{array}$ & 4.9 & 305 & - & 123 & No identified source & CT \\
\hline $\begin{array}{l}\text { El Braks et al. } \\
(2004)\end{array}$ & $71, \mathrm{~F}$ & Urinary incontinence & $\begin{array}{c}\text { Fever, sore } \\
\text { throat, and } \\
\text { epigastric pain }\end{array}$ & 1.6 & 521 & - & 73 & Pharyngitis & $\begin{array}{l}\text { US and } \\
\text { CT }\end{array}$ \\
\hline
\end{tabular}


TABLE 1: Continued.

\begin{tabular}{|c|c|c|c|c|c|c|c|c|c|}
\hline Author (year) & $\begin{array}{l}\text { Age, } \\
\text { Isex }\end{array}$ & Comorbidities & Symptoms & $\begin{array}{c}\text { Bilirubin } \\
(\mathrm{mg} / \mathrm{dl})\end{array}$ & $\begin{array}{l}\text { ALP } \\
(\mathrm{U} / \mathrm{L}) \\
\end{array}$ & $\begin{array}{c}\text { AST } \\
(\mathrm{U} / \mathrm{L}) \\
\end{array}$ & $\begin{array}{c}\text { ALT } \\
(\mathrm{U} / \mathrm{L}) \\
\end{array}$ & $\begin{array}{l}\text { Source of } \\
\text { infection }\end{array}$ & $\begin{array}{l}\text { Imaging } \\
\text { modality }\end{array}$ \\
\hline $\begin{array}{l}\text { Etienne et al. } \\
\text { (2001) }\end{array}$ & $68, \mathrm{M}$ & $\begin{array}{l}\text { TB and pulmonary } \\
\text { embolism (not on } \\
\text { anticoagulation) }\end{array}$ & Fever & - & - & - & - & $\begin{array}{c}\text { Possible } \\
\text { oropharyngeal source } \\
\text { and concomitant liver } \\
\text { abscess }\end{array}$ & CT \\
\hline $\begin{array}{l}\text { Schweigart } \\
\text { et al. (2005) }\end{array}$ & $67, \mathrm{M}$ & $\begin{array}{l}\mathrm{TB} \text {, stroke, } \mathrm{T} 2 \mathrm{DM} \text {, and } \\
\text { upper respiratory } \\
\text { infection weeks prior }\end{array}$ & $\begin{array}{l}\text { Fatigue, night } \\
\text { sweats, and } \\
\text { nausea }\end{array}$ & - & 175 & - & - & $\begin{array}{c}\text { Possible } \\
\text { oropharyngeal source }\end{array}$ & $\begin{array}{l}\text { CT and } \\
\text { MRI }\end{array}$ \\
\hline
\end{tabular}

Abbreviations: M: male, F: female, TB: tuberculosis, T2DM: type 2 diabetes mellitus, UC: ulcerative colitis, HNSCC: head and neck squamous cell cancer, COPD: chronic obstructive pulmonary disease, CAD: coronary artery disease, EtOH: alcohol, CM: cardiomyopathy, ALP: alkaline phosphatase, AST: asparatate aminotransferase, ALT: alanine aminotransferase, CT: computed tomography, US: ultrasound, MRI: magnetic resonance imaging, MRCP: magnetic resonance pancreaticography, NL: reported normal.

Pylephlebitis complications include thrombus extension into the superior mesenteric vein (SMV), thrombosis of the intrahepatic branches of the portal vein, liver abscesses, and inferior mesenteric vein thrombosis, in descending order of frequency [3]. Intestinal ischemia, a feared complication, can occur in $25 \%$ of patients with SMV thrombosis, carrying a mortality of $20 \%[3,29]$. Fortunately, the presence of a nonocclusive SMV thrombus in the reported case rendered the patient not at risk for intestinal ischemia; however, early recognition and treatment was critical to prevent progression. Long-term complications of untreated pylephlebitis include risks of chronic thrombus formation and resulting portal hypertension with associated complications of variceal bleeding and portal cholangiopathy [30].

Of the twenty-two cases of fusobacterium-related pylephlebitis, $41 \%$ involved solely the PV, 32\% involved some combination of the PV, SMV, IMV, or splenic vein, and $14 \%$ involved the SMV alone. There was isolated involvement of the right hepatic vein in two cases, and in only one case was the IMV solely affected (Table 2). The variability in thrombosis extent, variable anticoagulation, and follow-up periods render assessing whether thrombus extent affects long-term outcomes limited.

The mainstay treatment of pylephlebitis is fluid resuscitation, antibiotics, and often anticoagulation [4]. Antibiotic choices can be guided by culture and sensitivity when available or empirically with broad-spectrum enteric and anaerobic coverage $[20,21]$. Two large case series report similar mortality in patients treated with a combination of anticoagulation and antibiotics vs. antibiotics only $[4,5]$. However, anticoagulation lowers the risks of future chronic portal hypertension and, hence, is frequently utilized in the absence of contraindications [5]. The length of anticoagulation utilized is a minimum of 3 months, but the optimal duration is unclear [5]. Choices of anticoagulation include unfractionated heparin in the acute setting later transitioned to low molecular weight heparin, warfarin, or direct oral anticoagulants (DOAC) $[5,31]$. Treatment of Fusobacterium-spp.-associated pylephlebitis in the same vein follows the same principles. However, significant heterogeneity has been reported in use and duration of anticoagulation, where some authors anticoagulated patients only for the duration of their hospitalization while others anticoagulated patients between 3-9 months (Table 2). Given the absence of other risk factors for recurrent thrombosis in our patient, we elected to treat the event like a provoked deep vein thrombosis for a duration of 3 months.

Interestingly, while mortality has been reported as $11 \%$ in one case series of patients with pylephlebitis [4] and $12 \%$ in another [5], short-term outcome of patients with Fusobacterium-spp.-associated pylephlebitis appears more favorable with illness resolution in all cases where follow-up was reported (21 of 22 cases) and no cases of mortality including the reported case (Table 2). While this may represent a less fulminant course, it could be secondary to selection reporting biases that case reports are inherently prone to.

In conclusion, we present a case of pylephlebitis, a rare and often deadly complication, in the context of Fusobacterium necrophorum bacteremia. Our patient, whose presentation was mild and nonspecific, later developed septic shock. Adequate supportive therapy, prompt antibiotic therapy, and anticoagulation therapy with a DOAC for 3 months resulted in a favorable outcome. We hope that the presented case and review of existing cases contribute to the understanding of the subtle and insidious course of this rare complication. 
TAвLE 2: Thrombus extent, management, and outcomes in patients with Fusobacterium-spp.-associated pylephlebitis.

\begin{tabular}{|c|c|c|c|c|c|}
\hline Author (year) & Clot extent & Antibiotic regimen & $\begin{array}{l}\text { Anticoagulation } \\
\text { agent }\end{array}$ & $\begin{array}{l}\text { Anticoagulation } \\
\text { duration }\end{array}$ & Outcomes \\
\hline Presented case & PV and SMV & $\begin{array}{c}\text { I: vancomycin and } \\
\text { meropenem } \\
\text { F: ertapenem ( } 2 \text { weeks })\end{array}$ & $\begin{array}{l}\text { UFH and } \\
\text { apixaban }\end{array}$ & 3 months & $\begin{array}{c}\text { Recovery and elective } \\
\text { sigmoidectomy } 2 \text { years later }\end{array}$ \\
\hline $\begin{array}{l}\text { Abdallah et al. } \\
(2020)\end{array}$ & $\begin{array}{l}\text { PV, SMV, and } \\
\text { IMV }\end{array}$ & $\begin{array}{l}\text { I: meropenem } \\
\text { F: ertapenem (1 month) }\end{array}$ & UFH and warfarin & 6 months & $\begin{array}{l}\text { Recovery and asymptomatic } \\
\text { at } 6 \text {-month follow-up }\end{array}$ \\
\hline $\begin{array}{l}\text { Hamera et al. } \\
(2019)\end{array}$ & PV & $\begin{array}{c}\text { I: meropenem } \\
\text { F: ceftriaxone/metronidazole } \\
(1 \text { month })\end{array}$ & Enoxaparin & 1 month & $\begin{array}{l}\text { Recovery and no long-term } \\
\text { follow-up }\end{array}$ \\
\hline $\begin{array}{l}\text { Le Roux et al. } \\
\text { (2006) }\end{array}$ & SMV & $\begin{array}{c}\text { F: amoxicillin-clavulanate } \\
\text { and metronidazole (10 } \\
\text { months) }\end{array}$ & UFH & Hospitalization & $\begin{array}{c}\text { Recovery, } 10 \text {-month follow- } \\
\text { up, and abdominal US with } \\
\text { chronic PVT }\end{array}$ \\
\hline $\begin{array}{l}\text { Mellor et al. } \\
(2017)\end{array}$ & IMV & $\begin{array}{l}\text { I: piperacillin-tazobactam } \\
\text { F: ertapenem (1 month) }\end{array}$ & UFH & Hospitalization & $\begin{array}{l}\text { Recovery, } 1-\text { month follow- } \\
\text { up, and CT with PVT } \\
\text { resolution }\end{array}$ \\
\hline $\begin{array}{l}\text { Moore et al. } \\
(2016)\end{array}$ & Right PV & $\begin{array}{l}\text { I: piperacillin-tazobactam } \\
\text { F: metronidazole ( } 4 \text { weeks) }\end{array}$ & $\begin{array}{l}\text { Enoxaparin and } \\
\text { warfarin }\end{array}$ & 6 months & $\begin{array}{l}\text { Recovery and asymptomatic } \\
\text { at follow-up }\end{array}$ \\
\hline $\begin{array}{l}\text { Radovanovic } \\
\text { et al. (2019) }\end{array}$ & PV & $\begin{array}{l}\text { I: ceftriaxone/metronidazole } \\
\text { F: amoxicillin-clavulanate ( } 2 \\
\text { weeks) }\end{array}$ & $\begin{array}{l}\text { Enoxaparin and } \\
\text { warfarin }\end{array}$ & 3 months & $\begin{array}{l}\text { Recovery and asymptomatic } \\
\text { at } 1 \text {-month follow-up }\end{array}$ \\
\hline $\begin{array}{l}\text { Rahmati et al. } \\
\text { (2017) }\end{array}$ & PV & $\begin{array}{c}\text { I: ertapenem } \\
\text { F: ceftriaxone/metronidazole } \\
(2 \text { months), ampicillin- } \\
\text { clavulanate ( } 2 \text { months })\end{array}$ & Enoxaparin & - & $\begin{array}{l}\text { Recovery and 4-month } \\
\text { follow-up CT with } \\
\text { cavernous PV } \\
\text { transformation }\end{array}$ \\
\hline $\begin{array}{l}\text { Tharu and et al. } \\
(2020)\end{array}$ & $\begin{array}{l}\text { Superior right } \\
\text { hepatic vein }\end{array}$ & $\begin{array}{l}\text { I: vancomycin/ceftriaxone/ } \\
\text { metronidazole } \\
\text { F: ceftriaxone/metronidazole } \\
\text { (6 weeks) }\end{array}$ & None & - & $\begin{array}{l}\text { Recovery and 1-month CT } \\
\text { with PVT resolution }\end{array}$ \\
\hline $\begin{array}{l}\text { Akhrass et al. } \\
(2015)\end{array}$ & $\begin{array}{l}\text { Main PV at the } \\
\text { confluence }\end{array}$ & $\begin{array}{l}\text { I: piperacillin-tazobactam } \\
\text { F: Clindamycin ( } 6 \text { weeks) }\end{array}$ & UFH and warfarin & 6 weeks & $\begin{array}{l}\text { Recovery and follow-up not } \\
\text { reported }\end{array}$ \\
\hline $\begin{array}{l}\text { Zheng et al. } \\
\text { (2014) }\end{array}$ & $\begin{array}{l}\text { Right hepatic } \\
\text { vein }\end{array}$ & $\begin{array}{l}\text { I: cefepime } \\
\text { F: clindamycin }\end{array}$ & $\begin{array}{c}\text { Enoxaparin and } \\
\text { warfarin }\end{array}$ & - & $\begin{array}{l}\text { Recovery and 2-month } \\
\text { follow-up }\end{array}$ \\
\hline $\begin{array}{l}\text { Hamidi et al. } \\
(2008) \text { case } 1\end{array}$ & SMV & $\begin{array}{c}\text { I: amoxicillin-clavulanate } \\
\text { (during admission) } \\
\text { F: }-\end{array}$ & $\begin{array}{l}\text { LMWH and } \\
\text { fluindione }\end{array}$ & 6 months & $\begin{array}{c}\text { Recovery and 5-month } \\
\text { follow-up US with PVT } \\
\text { resolution }\end{array}$ \\
\hline $\begin{array}{l}\text { Hamidi et al. } \\
(2008) \text { case } 2\end{array}$ & SMV & None & None & None & Left against medical advice \\
\hline Soo et al. (1999) & $\mathrm{PV}$ andSMV & $\begin{array}{l}\text { I: ciprofloxacin/ } \\
\text { metronidazole/penicillin } \\
\text { F: amoxicillin-clavulanate/ } \\
\text { metronidazole (6 weeks) }\end{array}$ & UFH and warfarin & 6 months & $\begin{array}{l}\text { Recovery and 4-month MRI } \\
\text { with resolved PVT }\end{array}$ \\
\hline $\begin{array}{l}\text { Shahani et al. } \\
\text { (2011) }\end{array}$ & $\begin{array}{l}\text { Left and right } \\
\text { PV, SMV, and } \\
\text { splenic vein }\end{array}$ & $\begin{array}{l}\text { I: vancomycin/meropenem } \\
\text { F: tigecycline ( } 4 \text { weeks) }\end{array}$ & None & None & $\begin{array}{c}\text { Recovery and 2-month CT } \\
\text { with cavernous PV } \\
\text { transformation }\end{array}$ \\
\hline $\begin{array}{l}\text { Clarke et al. } \\
(2003)\end{array}$ & $\mathrm{PV}$ and SMV & $\begin{array}{c}\text { F: benzylpenicillin/ } \\
\text { metronidazole/ciprofloxacin } \\
\text { (6 weeks) }\end{array}$ & UFH and warfarin & Indefinite & $\begin{array}{l}\text { Recovery and } 7 \text {-weeks US } \\
\text { with PVT improvement }\end{array}$ \\
\hline $\begin{array}{l}\text { Redford et al. } \\
(2005)\end{array}$ & PV & $\begin{array}{c}\text { I: metronidazole/ } \\
\text { benzylpenicillin } \\
\text { F: clindamycin ( } 5 \text { weeks })\end{array}$ & $\begin{array}{l}\text { LMWH and } \\
\text { warfarin }\end{array}$ & 3 months & $\begin{array}{l}\text { Recovery and 3-months } \\
\text { follow-up }\end{array}$ \\
\hline $\begin{array}{l}\text { Bultink et al. } \\
\text { (1999) }\end{array}$ & PV & $\begin{array}{c}\text { I: imipenem } \\
\text { F: penicillin G (6 weeks) }\end{array}$ & IV heparin & Hospitalization & $\begin{array}{l}\text { Recovery and 2-month US } \\
\text { with chronic PVT }\end{array}$ \\
\hline $\begin{array}{l}\text { Verna et al. } \\
\text { (2004) }\end{array}$ & Left PV & F: clindamycin (2 weeks) & None & None & $\begin{array}{c}\text { Recovery and 6-month CT } \\
\text { with chronic PVT and left } \\
\text { hepatic atrophy }\end{array}$ \\
\hline $\begin{array}{l}\text { El Braks et al. } \\
(2004)\end{array}$ & $\mathrm{PV}$ and SMV & $\begin{array}{c}\text { F: piperacillin-tazobactam (2 } \\
\text { weeks) and ofloxacin ( } 3 \\
\text { weeks) }\end{array}$ & $\begin{array}{l}\text { UFH and } \\
\text { fluindione }\end{array}$ & 9 months & $\begin{array}{c}\text { Recovery and CT at follow- } \\
\text { up (not reported) with } \\
\text { chronic PVT }\end{array}$ \\
\hline
\end{tabular}


TABLE 2: Continued.

\begin{tabular}{lccccc}
\hline Author (year) & Clot extent & Antibiotic regimen & $\begin{array}{c}\text { Anticoagulation } \\
\text { agent }\end{array}$ & $\begin{array}{c}\text { Anticoagulation } \\
\text { duration }\end{array}$ & Outcomes \\
\hline $\begin{array}{l}\text { Etienne et al. } \\
(2001)\end{array}$ & PV & $\begin{array}{c}\text { F: cefotaxime and } \\
\text { metronidazole (2 weeks) and } \\
\text { metronidazole (2 weeks) }\end{array}$ & LMWH & 3 weeks & $\begin{array}{c}\text { Recovery and 5-week US } \\
\text { with resolution }\end{array}$ \\
\begin{tabular}{l}
$\begin{array}{l}\text { Schweigart et al. } \\
(2005)\end{array}$ \\
\hline
\end{tabular} & PV & F: clindamycin & Warfarin & Indefinitely & $\begin{array}{c}\text { Recovery and no long-term } \\
\text { follow-up }\end{array}$ \\
\hline
\end{tabular}

Abbreviations: I: initial regimen, F: final regimen, PV: portal vein, SMV: superior mesenteric vein, IMV: inferior mesenteric vein, CT: computed tomography, US: ultrasound, MRI: magnetic resonance imaging, UFH: unfractionated heparin, LMWH: low-molecular-weight heparin, PVT: portal vein thrombosis.

\section{Data Availability}

Data regarding this case/manuscript are available upon request from the corresponding author.

\section{Consent}

Informed consent was obtained from the patient for publication purposes.

\section{Disclosure}

The abstract of this paper was presented as a poster in the American College of Gastroenterology Meeting, Philadelphia, Pennsylvania, USA, 2018.

\section{Conflicts of Interest}

The authors report no conflicts of interest.

\section{Authors' Contributions}

N.L is the primary author, wrote the first draft of the manuscript, and performed the literature review with K.S who also participated in writing the first draft of the manuscript. Z.I, M.K, and I.H supervised the entire work and participated in manuscript drafting and editing.

\section{References}

[1] J. W. Dean, S. O. Trerotola, V. J. Harris, J. J. Snidow, and D. Hawes, "Percutaneous management of suppurative pylephlebitis," Journal of Vascular and Interventional Radiology, vol. 6, no. 4, pp. 585-588, 1995.

[2] E. J. Balthazar and P. Gollapudi, "Septic thrombophlebitis of the mesenteric and portal veins: CT imaging," Journal of Computer Assisted Tomography, vol. 24, no. 5, pp. 755-760, 2000.

[3] T. Kanellopoulou, A. Alexopoulou, G. Theodossiades, J. Koskinas, and A. J. Archimandritis, "Pylephlebitis: an overview of non-cirrhotic cases and factors related to outcome," Scandinavian Journal of Infectious Diseases, vol. 42, no. 11-12, pp. 804-811, 2010.

[4] A. J. Choudhry, Y. M. K. Baghdadi, M. A. Amr, M. J. Alzghari, D. H. Jenkins, and M. D. Zielinski, "Pylephlebitis: a review of 95 cases," Journal of Gastrointestinal Surgery, vol. 20, no. 3, pp. 656-661, 2016.

[5] L. Naymagon, D. Tremblay, T. Schiano, and J. Mascarenhas, "The role of anticoagulation in pylephlebitis: a retrospective examination of characteristics and outcomes," Journal of
Thrombosis and Thrombolysis, vol. 49, no. 2, pp. 325-331, 2020.

[6] D. Creemers-Schild, F. Gronthoud, L. Spanjaard, L. G. Visser, C. N. M. Brouwer, and E. J. Kuijper, "Fusobacterium necrophorum, an emerging pathogen of otogenic and paranasal infections?" New Microbes and New Infections, vol. 2, no. 3, pp. 52-57, 2014.

[7] T. Riordan, "Human infection with fusobacterium necrophorum (necrobacillosis), with a focus on Lemierre's syndrome," Clinical Microbiology Reviews, vol. 20, no. 4, pp. 622-659, 2007.

[8] L. Zheng and B. Giri, "Gastrointestinal variant of Lemierre syndrome," American Journal of Therapeutics, vol. 23, no. 3, pp. e933-e936, 2016.

[9] R. Soo, I. Gosbell, J. Gallo, and C. S. Pokorny, "Septic portal vein thrombosis due to Fusobacterium necrophorum," Australian and New Zealand Journal of Medicine, vol. 29, no. 4, pp. 569-570, 1999.

[10] L. Shahani and N. Khardori, "Fusobacterium necrophorumbeyond Lemierres syndrome," Case Reports, vol. 2011, p. bcr0720114527, 2011.

[11] J. H. Schweigart, A. Klotsas, S. Schelenz, and K. Dhatariya, "Portal vein thrombosis despite anticoagulation in a person with diabetes," Journal of the Royal Society of Medicine, vol. 98, no. 4, pp. 161-163, 2005.

[12] M. R. Redford, R. Ellis, and C. J. Rees, "Fusobacterium necrophorum infection associated with portal vein thrombosis," Journal of Medical Microbiology, vol. 54, no. 10, pp. 993-995, 2005.

[13] K. Hamidi, A. Pauwels, M. Bingen et al., "Thrombose veineuse mésentérico-porte aiguë associée à une septicémie à Fusobacterium necrophorum," Gastroentérologie Clinique et Biologique, vol. 32, no. 8-9, pp. 734-739, 2008.

[14] R. El Braks, F. Harnois, N. Boutros et al., "Mesenteric adenitis and portal vein thrombosis due to Fusobacterium nucleatum," European Journal of Gastroenterology \& Hepatology, vol. 16, no. 10, pp. 1063-1066, 2004.

[15] M. G. Clarke, N. J. Kennedy, and K. Kennedy, "Serious consequences of a sore throat," Annals of the Royal College of Surgeons of England, vol. 85, no. 4, pp. 242-244, 2003.

[16] F. A. Akhrass, L. Abdallah, S. Berger, and R. Sartawi, "Gastrointestinal variant of Lemierre's syndrome complicating ruptured appendicitis," IDCases, vol. 2, no. 3, pp. 72-76, 2015.

[17] B. Nigussie, D. Woredekal, F. I. Abaleka, M. Gizaw, and B. Tharu, "A sporadic case of disseminated fusobacterium causing pylephlebitis and intracranial and hepatic abscesses in a healthy young patient," Cureus, vol. 12, no. 7, 2020.

[18] J. A. Moore and S. Rambally, "Fusobacterium nucleatum bacteremia presenting with portal vein thrombosis: an abdominal Lemierre syndrome?" The American Journal of Medicine, vol. 130, no. 6, pp. e255-e256, 2017. 
[19] K. Le Roux, P. Sève, E. Gomard et al., "Un variant du syndrome de Lemierre: abcès hépatiques et thrombose veineuse sus-hépatique secondaire à une septicémie à Fusobacterium nucleatum," La Revue de Médecine Interne, vol. 27, no. 6, pp. 482-486, 2006.

[20] M. Abdallah, A. Gohar, S. Naryana Gowda, H. M. Abdullah, and A. Al-hajjaj, "Pylephlebitis associated with inferior mesenteric vein thrombosis treated successfully with anticoagulation and antibiotics in a 37-year-old male," Case Reports in Gastrointestinal Medicine, vol. 20203 pages, 2020.

[21] S. Pérez-Bru, C. Nofuentes-Riera, A. García-Marín, P. LuriPrieto, M. Morales-Calderón, and S. García-García, "Pileflebitis: una extraña pero posible complicación de las infecciones intraabdominales," Cirugía Y Cirujanos, vol. 83, no. 6, pp. 501-505, 2015.

[22] T. E. Mellor, N. Mitchell, and J. Logan, "Lemierre's syndrome variant of the gut," BMJ Case Reports, vol. 2017, 2017.

[23] M. Etienne, I. Gueit, P. Abboud, J.-L. Pons, S. Jacquot, and F. Caron, "Fusobacterium nucleatum hepatic abscess with pylephlebitis associated with idiopathic $\mathrm{CD}_{4}{ }^{+} \mathrm{T}$ lymphocytopenia," Clinical Infectious Diseases, vol. 32, no. 2, pp. 326-328, 2001.

[24] E. C. Verna, A. Larghi, S. G. Faddoul, J. A. Stein, and H. J. Worman, "Portal vein thrombosis associated with fusobacterium nucleatum septicemia in a patient with ulcerative colitis," Journal of Clinical Gastroenterology, vol. 38, no. 7, pp. 611-612, 2004.

[25] I. E. M. Bultink, J. W. Dorigo-Zetsma, M. G. Koopman, and E. J. Kuijper, "Fusobacterium nucleatumSepticemia and portal vein thrombosis," Clinical Infectious Diseases, vol. 28, no. 6, pp. 1325-1326, 1999.

[26] N. Radovanovic, I. Dumic, M. Veselinovic et al., "Fusobacterium necrophorum subsp. necrophorum liver abscess with pylephlebitis: an abdominal variant of Lemierre's syndrome," Case Reports in Infectious Diseases, vol. 20206 pages, 2020.

[27] E. Rahmati, R. C. She, B. Kazmierski, P. J. Geiseler, and D. Wong, "A case of liver abscess and fusobacterium septicemia," IDCases, vol. 9, pp. 98-100, 2017.

[28] W.-K. Lee, S. D. Chang, V. A. Duddalwar et al., "Imaging assessment of congenital and acquired abnormalities of the portal venous system," Radiographics, vol. 31, no. 4, pp. 905-926, 2011.

[29] S. Acosta, A. Alhadad, P. Svensson, and O. Ekberg, "Epidemiology, risk and prognostic factors in mesenteric venous thrombosis," British Journal of Surgery, vol. 95, no. 10, pp. 1245-1251, 2008.

[30] J. Hoekstra and H. L. A. Janssen, "Vascular liver disorders (II): portal vein thrombosis," Netherlands Journal of Medicine, vol. 67, no. 2, 2009.

[31] P. Priyanka, J. T. Kupec, M. Krafft, N. A. Shah, and G. J. Reynolds, "Newer oral anticoagulants in the treatment of acute portal vein thrombosis in patients with and without cirrhosis," International Journal of Hepatology, vol. 2018, 9 pages, 2018. 